Fixed Point Theory, 21(2020), No. 1, 191-210

DOI: $10.24193 /$ fpt-ro.2020.1.14

http://www.math.ubbcluj.ro/ nodeacj/sfptcj.html

\title{
DISCUSSION ON THE EXISTENCE OF BEST PROXIMITY POINTS IN METRIC SPACES
}

\author{
SHIHUANG HONG*, JIE ZHOU*, JI CHEN*, HAIYANG HOU* AND LI WANG** \\ * Science College, Hangzhou Dianzi University, \\ Hangzhou, 310018, People's Republic of China \\ *** Mathematical Department, Hainan University, Haikou, 570228, \\ People's Republic of China \\ E-mail: hongshh@hotmail.com
}

\begin{abstract}
In this paper, we modify the definition of some generalized proximal contractions and enumerate a list of equivalent conditions for various versions of generalized proximal contractions of non-self set-valued mappings on (ordered) metric spaces. By using the fixed point means, we establish the existence of best proximity points for mappings involving such contractions which extend and improve many existing related results, as well as, reveal that most of existing best proximity point theorems on metric spaces are in fact equivalent and immediate consequences of well-known fixed point theorems. Finally, some examples are given to support our results.

Key Words and Phrases: Fixed points, best proximity points, generalized contractions, $\alpha-$ proximal admissible mappings.
\end{abstract}

2010 Mathematics Subject Classification: 37C25, 47H10.

Funding. This study was supported by National Natural Science Foundation of China (71771068, 71471051).

\section{REFERENCES}

[1] A. Abkar, M. Gabeleh, The existence of best proximity points for multivalued non-self-mappings, Revista Real Acad. Ciencias Exactas, Físicas y Naturales. Serie A. Mat., 107(2013), 319-325.

[2] A. Abkar, M. Gabeleh, A note on some best proximity point theorems proved under P-property, Abstr. Appl. Anal., 2013(2013), DOI: 10.1155/2013/189567.

[3] M. Alghamdi, N. Shahzad, F. Vetro, Best proximity points for some classes of proximal contractions, Abstr. Appl. Anal., 2013(2013), Art. ID 713252, 10 pages.

[4] M.U. Ali, T. Kamaran, N. Shahzad, Best proximity point for $\alpha-\psi$-proximal contractive multimap, Abstr. Appl. Anal., 2014(2014), Art. ID 181598.

[5] A. Almeida, J. Harjani, K. Sadarangani, Existence and uniqueness of best proximity point for contractions of Geraghty type, Revista Real Acad. Ciencias Exactas, Físicas y Naturales. Serie A. Mat., 108(2014), 957-971.

[6] A. Amini-Harandi, Best proximity points for proximal generalized contractions in metric spaces, Optim. Lett., 7 (2013), 913-921.

[7] S. Banach, Sur les opérations dans les ensembles abstraits et leurs applications aux équations intégrales, Fundam. Math., 3(1922), 133-181.

Supported by Natural Science Foundation of China $(71771068,71471051)$. 
[8] S.S. Basha, Best proximity point theorems generalizing the contraction principle, Nonlinear Anal., 74(2011), 5844-5850.

[9] S.S. Basha, Best proximity points: Optimal solutions, J. Optim. Theory Appl., 151(2011), 210-216.

[10] S.S. Basha, Best proximity point theorems, J. Approx. Theory, 163(2011), 1772-1781.

[11] S.S. Basha, Best proximity point theorems: An exploration of a common solution to approximation and optimization problem, Appl. Math. Comput., 218(2012), 9773-9780.

[12] S.S. Basha, Best proximity theorems on partially ordered sets, Optim. Lett., 7(2013), 1035-1043.

[13] S.S. Basha, Best proximity point theorems: unriddling a special nonlinear programming problem, TOP, 22(2014), 543-553.

[14] S.S. Basha, N. Shahzad, Best proximity point theorems for generalized proximal contractions, Fixed Point Theory Appl., 2012(2012), no. 42.

[15] S. Cho, Best proximity points for generalized almost-Geraghty type contractions in partially ordered metric spaces, Appl. Math. Sci., 63 (2015), no. 9, 3143-3154.

[16] B.S. Choudhury, P. Maity, P. Konar, A global optimality result using nonself mappings, OPSEARCH, 51(2014), no. 2, 312-320.

[17] A. Fernández-León, Best proximity points for proximal contractions, Math. Funct. Anal., 18(2012), 1-10.

[18] M. Gabeleh, Proximal weakly contractive and proximal nonexpansive non-self-mappings in metric and Banach spaces, J. Optim. Theory Appl., 158(2013), no. 2, 615-625.

[19] M. Gabeleh, Best proximity points: global minimization of multivalued non-self mappings, Optim. Lett., 8(2014), 1101-1112.

[20] M. Gabeleh, Best proximity point theorems for single- and set-valued non-self mappings, Acta Math. Sci., 34(2014), no. 5, 1661-1669.

[21] M. Gabeleh, Best proximity point theorems via proximal non-self mappings, J. Optim Theory Appl., 164(2015), 565-576.

[22] M. Gabeleh, Best proximity points for weak proximal contractions, Bull. Malays. Math. Sci. Soc., 38(2015), no. 1, 143-154.

[23] S.H. Hong, D. Guan, L. Wang, Hybrid fixed points of multivalued operators in metric spaces with applications, Nonlinear Anal., 70(2009), 4106-4117.

[24] J. Jachymski, Equivalent conditions for generalized contraction on (ordered) metric spaces, Nonlinear Anal., 74(2011), 768-774.

[25] M. Jleli, E. Karapinar, A short note on the equivalence between best proximity points and fixed point results, J. Ineq. Appl., 2014(2014), no. 246.

[26] M. Jleli, E. Karapinar, B. Samet, Best proximity points for generalized $\alpha$ - $\psi$-proximal contractive type mappings, J. Appl. Math., 2013 (2013), Art. ID 534127, 10 pages.

[27] M. Jleli, E. Karapinar, B. Samet, On best proximity points under the P-property on partially ordered metric spaces, Abstr. Appl. Anal., 2013(2013), Art. ID 150970, 6 pages.

[28] M. Jleli, B. Samet, Best proximity points for $\alpha-\psi$-proximal contractive type mappings and applications, Bull. Sci. Math., 137(2013), 977-995.

[29] E. Karapinar, V. Pragadeeswarar, M. Marudai, Best proximity point for generalized proximal weak contractions in complete metric space, J. Appl. Math., 2014(2014), Art. ID 150941, 6 pages.

[30] S.B. Nadler, Multi-valued contraction mappings, Pacific J. Math., 30(1969), 475-488.

[31] M. Omidvari, S. Vaezpour, R. Saadati, S. Lee, Best proximity point theorems with Suzuki distances, J. Ineq. Appl., 2015(2015), no. 27.

[32] V. Pragadeeswarar, M. Marudai, Best proximity points for generalized proximal weak contractions in partially ordered metric spaces, Optim. Lett., 9(2015), 105-118.

[33] V. Pragadeeswarar, M. Marudai, Best proximity points: approximation and optimization in partially ordered metric spaces, Optim. Lett., 7(2013), 1883-1892.

[34] P. Salimi, P. Vetro, A best proximity point theorem for generalized Geraghty-Suzuki contractions, Bull. Malays. Math. Sci. Soc., 39(2016), 245-256.

[35] P. Samet, A. Latif, N. Hussain, Modified $\alpha-\psi$-contractive mappings with applications, Fixed Point Theory Appl., 2013(2013), 151. 
[36] W. Sanhan, C. Mongkolkeha, P. Kumam, Generalized proximal $\psi$-contraction mappings and best proximity points, Abstr. Appl. Anal., 2012(2012), 19 pages, doi:10.1155/2012/896912.

[37] R.V. Sankar, A best proximity point theorem for weakly contractive non-self-mappings, Nonlinear Anal., 74(2011), 4804-4808.

[38] S.P. Singh, B.A. Meede, On common fixed point theorems, Bull. Austral. Math. Soc., 16(1977), 49-53.

[39] A. Sultana, V. Vetrivel, On the existence of best proximity points for generalized contractions, Appl. Gen. Topol., 15(2014), no. 1, 55-63.

Received: November 28, 2016; Accepted: March 12, 2019. 